\title{
Candidate gene association studies: a comprehensive guide to useful in silico tools
}

\author{
Radhika Patnala, Judith Clements and Jyotsna Batra
}

\begin{abstract}
The candidate gene approach has been a pioneer in the field of genetic epidemiology, identifying risk alleles and their association with clinical traits. With the advent of rapidly changing technology, there has been an explosion of in silico tools available to researchers, giving them fast, efficient resources and reliable strategies important to find casual gene variants for candidate or genome wide association studies (GWAS). In this review, following a description of candidate gene prioritisation, we summarise the approaches to single nucleotide polymorphism (SNP) prioritisation and discuss the tools available to assess functional relevance of the risk variant with consideration to its genomic location. The strategy and the tools discussed are applicable to any study investigating genetic risk factors associated with a particular disease. Some of the tools are also applicable for the functional validation of variants relevant to the era of GWAS and next generation sequencing (NGS).
\end{abstract}

Keywords: Candidate gene, SNP, LD, In-silico, Association studies, Cancer

\section{Review \\ Introduction}

Candidate gene studies have been at the forefront of genetic association studies i.e. identifying risk variants associated with a particular disease. Candidate gene studies are relatively cheap and quick to perform, and are focused on the selection of genes that have been in some way related to the disease previously and thus come with prior knowledge about gene function. The candidate gene approach begins with selection of a putative candidate gene based on its relevance in the mechanism of the disease (trait) being investigated [1]. This is followed by assessing and selecting polymorphisms, usually the tag Single NucleotidePolymorphim (SNPs) (described later in this review) and/or having a functional consequence, either by affecting gene regulation or its protein product $[1,2]$. Finally, the gene variant is verified for disease (trait) association by observing its occurrence in random test subjects (cases) having the disease and the selected control subjects which do not; and is then evaluated for its association with disease prognosis and diagnosis and its future potential as a biomarker. This makes the knowledge derived from

\footnotetext{
* Correspondence: jyotsna.batra@qut.edu.au
Australian Prostate Cancer Research Centre - Queensland, Institute of Health

* Correspondence: jyotsna.batra@qut.edu.au and Biomedical Innovation, Queensland University of Technology, Brisbane, QLD 4059, Australia
}

(c) 2013 Patnala et al.; licensee BioMed Central Ltd. This is an Open Access article distributed under the terms of the Creative Commons Attribution License (http://creativecommons.org/licenses/by/2.0), which permits unrestricted use, distribution, and reproduction in any medium, provided the original work is properly cited. candidate gene studies valuable and clinically relevant as a medicine initiatives in future treatments of genetic disorders [3].

Candidate gene association studies have been criticised on some aspects, but these can be duly overcome by the range of new tools and resources developed to this end. One such aspect is non-replication of results. One of the major issues for non-replication of the results involves population stratification, which can easily be circumvented by considering a replication study using an independent and random cohort of test and control populations, which reduces the chance of occurrence of a similar admixture showing similar patterns of variations [4]. The many collaborative projects taking place in recent years, such as the international HapMap project (http:// hapmap.ncbi.nlm.nih.gov/) [5] and 1000 genomes project (http://www.1000genomes.org/) [6], provide researchers with allele frequencies of SNPs and their correlation pattern (haplotypes) to analyse population stratification before pooling data from different populations. Another aspect is the mild uncertainty about if the results portray disease susceptibility of a common variant, or do they just represent certain ancestral differences existing by chance between the mixes of test or control populations. Additionally, the multiple comparisons issue due to 
accounting for the same SNP in various tests can lead to false discovery rates. This can be addressed in two ways, first by computing Bonferroni adjustments of the significance criterion (alpha) according to the number of genes/ SNPs/haplotypes (described later in this review) examined and second by performing permutation analysis of the association with allelic variation in the associating haplotype block. Although some argue that candidate gene studies must still meet statistical criteria for genome-wide significance, such a conservative threshold seems overly stringent, particularly in the context of a disorder with no (known) major gene effects. One of the other reasons for identifying a number of false positive findings could involve systemic genotyping errors, lack of statistical power due to smaller samples. In other instances, false negative findings (type II error) could be the reason for nonreplication $[7,8]$. False negative findings can be attributed to under evaluation of gene-gene interactions and gene environment interactions [7] and/or because of missing some causative polymorphisms during linkage disequilibrium (LD) considerations [9]. In silico initiatives which take into account LD and compile tag SNPs and haplotypes can be very helpful in circumventing this.

Considering these aspects along with cumulative effect of multiple loci and complex disease heterogeneity, a fine tuning of the candidate gene approach has been sorted after $[8,9]$. Completion of the first phase of the 1000 genome sequencing project has further provided new avenues for reconsidering candidate gene association approaches to dissect the complexity of many genetic disorders. One major step in this regard could be a careful and thorough selection of candidate genes and variations forming the basis towards association-analysis. This support is available through various targeted in silico tools to evaluate all aspects of the candidate gene and the prioritised SNPs in a strategic manner.

This review intends to summarize current bioinformatics tools and literature available for the purpose of selecting a candidate gene for disease association studies and the genetic variants such as SNPs, from these candidate genes de novo, or from within a linkage peak. Some of these in silico methods are applicable to the functional analysis of data generated through various candidate gene association studies or for variants identified through postGWAS fine mapping studies and/or next-generation sequencing.

\section{Selection of a candidate gene and retrieval of relevant sequence information}

Recent data mining software advancements have catered substantially to growing research needs making it much easier to cope with the initial phase of searching through the enormous amounts of literature present online and keeping constantly updated in order to intelligently select a candidate gene. Tools provided by iHOP Web services (http://www.ihop-net.org/UniPub/iHOP/) [10] enables general literature mining and PubCrawler (http:// pubcrawler.gen.tcd.ie/) [11] enables keeping track of daily updates. A commercial tool from Biovista - BioLab Experiment Assistant (http://www.biovista.com/bea/) [12] is uniquely designed around providing the user search capabilities to find concepts of interest (such as drugs, genes, molecules etc.) and review their interconnections visually, facilitating fine tuning of research strategy before getting down to reading literature. Ingenuity ${ }^{\circledR}$ Knowledge Base (http://www.ingenuity.com) is a depot of manually reviewed, enriched and sorted information of biological interactions and functional annotations, provided to the user through powered products like IPA ${ }^{\circledR}$, iReport ${ }^{\circledR}$ in a contextual interface, also linking out to the original articles.

Analysing participating pathways is an important aspect of any gene's functional analysis strategy. In this view, REACTOME (http://www.reactome.org) [13] is a cross referenced, manually curated and peer reviewed pathway database. LitInspector (http://www.litinspector.org) [14] and NetPath (http://www.netpath.org/index.html) [15] allow one to access curated signal transduction related literature and interaction pathways respectively. Predictive Networks (http://predictivenetworks.org/) [16] integrates gene interactions and networks information from PubMed literature and other online biological databases and presents it in an accessible and efficient user interface. Two other noteworthy commercial tools are GeneGo and Ingenuity IPA. GeneGo (http://www.genego.com/) technology facilitates pathway analysis to find interacting molecules and subsequent interactions relevant to the investigated trait or disease. Ingenuity IPA also considers pathway analysis in its package. Another website Topp Gene suite (http://toppgene.cchmc.org/) [17], provides tools for functional enrichment of genes based on a training gene set (to be provided by user), and also for including protein networks and neighbouring genes of the locus in analysis.

Finding candidate genes for further investigation, also defined as gene prioritisation has been covered in detail elsewhere [18]. The hosted web portal - Gene Prioritization Portal (http://www.esat.kuleuven.be/gpp), links out to 33 current computational tools for this purpose, such as GeneRank, GeneWanderer, Caesar, SNPs3D and GeneDistiller among many others. This resource compares many online computational tools and thus, provides an efficient and comprehensive guide to help the user develop a suitable gene prioritisation strategy, and is highly recommended.

Functional analysis of a gene is incomplete without a brief investigation for any existing disease associations. $\mathrm{OMIM}^{\circledR}$, Online Mendelian Inheritance in $\mathrm{Man}^{\circledR}$ (http://www.ncbi.nlm.nih.gov/omim) [19] is a database 
helpful to establish and/or investigate disease associations of gene of interest as it aims to lists all known genotype to phenotype correlations. PhenoPred (http:// www.phenopred.org/) [20] is another useful starting resource for crosschecking for gene-disease association to set the stage and establishing a gene's clinical relevance. An example of a disease specific web tool is Oncomine (https://www.oncomine.org) [21] providing an elaborate resource to cancer biologists interested in accessing cancer transcriptome data from large number of datasets collected, standardised and analysed as part of the Oncomine project. An elaborate description, comparison and usage strategy of tools currently available for the purpose of in silico gene function prediction relevant to cancer study, with their efficacy in suitably classifying uncharacterised cancer genes based on current knowledge from online databases has been given by $\mathrm{Hu}$ et al. [22].

Once a thorough assessment of literature and a holistic view of interacting pathways to the gene of interest have been considered, one is ready to focus on the gene composition and sequence. A gene locus can be analysed for various attributes. Many popular, consistently well updated and publically available databases provide genetic and functional information of a gene and its locus, which are advantageous to consider during SNP selection. Prior knowledge of the gene's functional and structural elements within and those in its periphery can elicit a better understanding of the putative function of the gene variants. Comprehensive sites such as Entrez Gene (http://www. ncbi.nlm.nih.gov/Entrez) [23] and Ensembl (http://www. ensembl.org) [24] host an organised, collective resource linking out to various tools providing general information on gene structure, expression, splice variants encoded proteins, regulatory elements, SNPs and the like. Assessing splicing variants is of extreme importance when dealing with eukaryotic genomes, primarily due to their direct relation with candidate gene transcription, and also the acute sensitivity of splicing sites to SNP variations. Such an example has been elucidated in our recent study of the Kallikrein15 (KLK15) gene locus, where a SNP (rs266851) closely located (15 kb downstream) to a novel exon, renders increased susceptibility to ovarian cancer survival and is predicted to play a role in alternative mRNA splicing [25]. Another incidence is of a fairly common intronic KLF6 gene polymorphism, called IVS1 $-27 \mathrm{G}>\mathrm{A}$, i.e. the IVS $\triangle A$ allele giving rise to an additional DNA binding site and increased expression of three alternative spliced transcripts of the gene [26]. Aceview (www.ncbi. nlm.nih.gov/IEB/Research/Acembly/) [27] provides an extensive annotated evaluation of cDNA supported transcriptome complete with data on mRNA and existing splice variants in the genome. The UCSC Genome Browser (http://genome.ucsc.edu/) [28] is an extremely efficient and popular tool, and extends to gauging genetic sequence information of gene loci in much detail. It provides, in numerous tracks, options to view transcript variants, repeats, evolutionary conservation and many other genetic modules which might be present in the gene of interest, and are relevant to the candidate SNPs under investigation. It also links to The Encyclopedia of DNA elements (ENCODE) (http://genome.ucsc.edu/ENCODE/) [29] which is a regularly updated database of functional and regulatory elements as found in the human and mouse genomes.

To assist in probing the functional importance of candidate genes while prioritising them, the VISTA Genome Browser (http://genome.lbl.gov/vista) [30] gives tools to compare your sequence with curated whole genome assemblies for regulatory elements and transcription factor binding sites (TFBS). It also links to VISTA Enhancer browser (http://enhancer.lbl.gov/) [31], which is a database of experimentally validated human enhancer elements, and to VISTA Region viewer (http://rviewer.lbl. gov/) [32], a tool for prioritising genomic regions for further studies. Another recommended tool is VarioWatch (Previously GenoWatch) (http://genepipe.ncgm.sinica.edu. tw/variowatch/main.do) [33] which retrieves comprehensive gene information in a particular region, in real time from various primary sources.

Various high-end computational resources developed in the last decade are freely available online and are updated continuously, although some limitations exist. Tools which rely on Gene Ontology (GO) to characterise information are limited because the GO annotation is an ongoing process, and cannot provide a full picture. Also, it shows a bias towards well known, better characterised diseases and research terms, hence, making the search miss on what could otherwise be functionally relevant to the gene under investigation [34]. Thus tools which support descriptive keyword search to identify desired genes are more useful in some cases. Care should be taken to use the most updated versions of tools available online, as these have been fine tuned to have better accuracy rates, are also backed by latest database resources such as the 1000 genomes project (in relation to genomic variants) and the latest genome assembly which is currently GRCh37/hg19 which can be tracked for updates at the Genome Reference Consortium website (http://www.ncbi.nlm. nih.gov/projects/genome/assembly/grc/).

A consolidated account of all the above mentioned resources is provided in Additional file 1: Table S1.

\section{Cataloguing SNPs in a candidate gene}

Once the relevance of the candidate gene and the spread of its functional elements (enhancer, promoter, intron, exon, UTR etc.) have been noted, the next step is cataloguing the SNPs present in the candidate gene, in its regulatory sequences (Promoter, TF binding sites, non coding 
regions), and in its surrounding regions which might have long distance effects on the gene function (eg. enhancers). A demonstration of this step and its context can be found in a recent review by us focussed on cataloguing all SNPs important to the Kallikrein gene locus [35].

Some resources which facilitate scouring the gene locus for submitted variants have been reviewed extensively by Coassin et al. [36] such as NCBI's dbSNP (http://www. ncbi.nlm.nih.gov/snp) [37,38]. PolyScan (http://genome. wustl.edu/pub/software/polyscan/) [39] can be used to reprocess the results to improve detection. BioQ (http:// bioq.saclab.net/) [40] enables one to track back to the experimental process flow and data source of the variant data. dbSNP-Q (https://cgsmd.isi.edu/dbsnpq/) [41] provides a downloadable interface which can be used to meaningfully analyse dbSNP data with custom designed tables which use task based queries to select and display relevant information. SNPper (http://snpper.chip.org/) [42], one of the tools provided at the CHIP bioinformatic tools website enables retrieval of SNP based on name or gene association and compliments it with additional useful tools such as FlankXtender to include sites flanking the gene. Evaluating functional elements in the genome for putative variations can be performed by RAVEN (Regulatory Analysis of Variation in ENhancers) (http://www. cisreg.ca/) although the link is not currently functional [43]. This is a web application specially designed to identify genetic variations in cis regulatory elements of the candidate gene through combining consideration of transcription, TFBS prediction and phylogenetic footprinting, enabling researchers to isolate SNPs which might have a direct consequence on transcriptional regulation of the genomic site [43]. A database providing sequenced and genotyped SNPs in genes implicated in cancer studies is the SNP500Cancer (http://variantgps.nci.nih.gov/cgfseq/ pages/snp500.do) [44] hosted by the Variant GPS (http:// variantgps.nci.nih.gov/cgfseq/pages/home.do). ANNOVAR (http://www.openbioinformatics.org/annovar/) [45] enables mining through the data from high throughput experiments and identifying, sorting, and prioritising candidate SNPs (variants) in important genomic regions in its filter based annotation. The SNPinfo Web Server (http:// snpinfo.niehs.nih.gov/) [46] provides many efficient, comprehensive and user friendly tools suited for various purposes such as GenePipe (for Candidate gene selection), GenomePipe (Functional SNP selection), LinkagePipe (SNP selection in one genomic loci of interest), TagSNP, FuncPred (querying SNP function prediction) and SNPseq (viewing SNPs in their genomic region context, with information on CpG sites), making this a one stop website for initial SNP investigation from scratch. All mentioned tools, which can be used for SNP cataloguing, are detailed in Additional file 1: Table S1; which also shows schematically the tools available and places the important step of choosing an SNP of interest in the context of candidate gene association studies.

\section{Selection of the tag SNPs for the association studies}

Linkage disequilibrium is a phenomenon where alleles associate at different loci non-randomly; carrying with them conserved combinations of SNPs. The most widely recognised measure for LD is $r^{2}$, where $\mathrm{r}$ is the correlation coefficient between two loci with alleles in association [47]. A gene locus hosting SNPs demonstrating LD have a higher propensity to be conserved in populations with recombination occurring on either side of it [48]. Analysis of LD within the candidate SNPs is a valued way of narrowing down on the limits of the disease susceptible genomic region [48]; because they will mostly be inherited together and show similar frequencies in affected individuals of a population. Such SNPs closely linked with each other and demonstrating LD effects can be tagged and represented by selected SNPs among them, referred to as tag SNPs. LD and its evolutionary and medical importance has been described in detail in several reviews $[48,49]$. Recently developed resources specific to LD analysis are, DistiLD http://distild.jensenlab.org/ [50], GLIDERS (http://www.sanger.ac.uk/resources/software/gliders/) [51], SNPAnalyser 2.0 (http://snp.istech21.com/snpanalyzer/2.0/) [52] further elaborated in Additional file 1: Table S1. SNAP (SNP Annotation and Proxy Search; http://www.broad.mit. $\mathrm{edu} / \mathrm{mpg} / \mathrm{snap} /$ ) [53] further includes data from the 1000 genomes project in its data pool and also provides graphical representations of regional LD analysis. A web link to multiple LD tools can be found at http://www.genes.org.uk/ software/LD-software.shtml [54].

Haplotypes can be defined as evolutionary conserved segments of DNA inherited together. It is at these regions that tag SNPs and LD effects are observed; such that genotyping one SNP in a locus can determine the effects of many others. The international HapMap project (http://hapmap.ncbi.nlm.nih.gov/) took the initiative of genotyping sections of human populations worldwide to bring the haplotype map, and accelerate the search for Haplotypes and tag SNPs to narrow down on statistically significant, reviewed disease associated loci, while understanding the patterns of genetic distribution in humans from diverse regions [5]. It currently provides this data to allow further analysis and interpretation of GWAS results with the use of imputation. A resource like Haploview (www.broad.mit.edu/mpg/haploview/) [55] takes its data resource from HapMap project and can assist greatly in LD analysis during gene and SNP prioritisation.

Following the identification of candidate SNPs and a peripheral analysis of their location in the genome, focussed computational tools designed to specifically understand downstream effects of SNPs depending on their 
genomic context and placement can be considered, thus, enabling in depth in silico analysis of the respective functional changes they might bring in cellular processes.

\section{Selection of candidate SNPs through function prediction} SNPs are classified according to their location in the gene locus, which also most times dictates the functional downstream effects of the SNP [56] and will guide the selection of appropriate computational tools towards its analysis. SNPs within the coding region of the gene but not causing any change in the formed protein, such that both alleles still encode the same protein sequence, are classified as synonymous SNPs. This is possible due to the degeneracy of the genetic code; and it does not cause any direct functional defects than from probable splicing variations. SNPs in the coding region which leads to a change in the translated amino acids and thus in the encoded protein are categorised as non-synonymous SNPs (nsSNPs), as encoded protein sequences differ between both alleles. While the functional role of non-synonymous SNPs is relatively straight forward, SNPs located in regulatory and intronic regions have recently gained importance upon recognition of their potential to deregulate transcriptional efficiency, gene expression and splicing [57-60]. Especially SNPs in regions encoding microRNA and non-coding RNAs can thus be considered for association studies [61]. An interesting tool to use in the start is the Variant Effect Predictor (http://www.ensembl.org/info/docs/variation/vep/index.html) [62] found within the Ensemble periphery which predicts the functional effect of known and unknown variants. Given below are detailed web tools specific to analyse SNPs in coding regions and in regulatory regions.

\section{Functional SNPs within the coding regions}

A nsSNP affect protein sequence and structure, and can affect its functionality depending on the position of the change and the amino acid it replaces. Usually such changes differ in the degree of deleterious effect they cause, with highly deleterious SNPs already being filtered out by nature through natural selection [63]. Thus, all nsSNPs discovered through high throughput studies, can be those with long ranging clinical implications to disease causation, and even though occurring in low frequency, are none the less quite important. For example, rs17632542 in the $K L K 3$ gene is implicated in high association to prostate cancer susceptibility, and rs1126497 with a $\mathrm{C} / \mathrm{T}$ polymorphism in epithelial cell adhesion molecule $(E p C A M)$ in its exon 3 has been linked to increased risk of breast cancer in chinese populations [64]. The knowledge of gain or loss of function attributed to a protein by the incidence of a nsSNP can be acquired by further functional analysis and experimental analysis. Analysis can begin with annotating the resulting protein sequence and structure of the variant carrying the SNP. Then subsequent investigation into functional aspects such as its conformation, enzymatic sites and amino acid interactions will reveal how the variation affects protein structure and function of the resultant phenotype. Additional file 2: Table S2 lists useful resources in this area.

Annotation of protein structure can be performed by resources such as SNPs3D (http://www.snps3d.org/) [65]. LS-SNP/PDB (http://ls-snp.icm.jhu.edu/ls-snp-pdb/) [66] lets one map the variations on $3 \mathrm{D}$ structures available in Protein Data Bank. ModBase (http://modbase.compbio. ucsf.edu) [67] goes one step further allowing comparative annotated protein structure models, also linking out to functional analysis of the SNP effect on protein. Data from various algorithms and functional criteria applied to the dbSNP dataset have been integrated by PolyDoms (http:// polydoms.cchmc.org) [68] to predict structural and functional protein variations, also integrating data on pathways, interactions and allelic variations from various sources [34]. UniProt (http://www.uniprot.org/) [69] provides a database for protein information while the direct SNP effect on protein function can be studied using SNPeffect (http://snpeffect.switchlab.org/) [70], and Pupasuite (http://pupasuite.bioinfo.cipf.es/) [71]. These, apart from providing other tools as discussed later, annotate protein structures and facilitate checking the protein for functional sites such as catalytic sites, DNA and protein binding sites and also those harbouring post translational modifications ([34] and references therein). Users should note that knowing the methodology used by the software is imperative to assess the accuracy and its relevance to the case being investigated [36].

Recent advancements in forecasting the effects of amino acid substitutions in protein sequence train computational tools to learn and then predict downstream effects of protein variants. These programs are trained by using either disease-associated alleles in databases or by experimentally varying amino acid substitutions to check for functional changes [34]. Many recent tools have been described and compared in the review by Mah et al. [72], albeit in a different mode of classification. Mah et al. [73] classify available algorithms as sequence versus structure based approaches, detailing advantage and drawbacks to both. The sequence-based approach uses induction of single base substitutions to predict effects on the function of resulting proteins [74], for example as PoPMuSiC (http:// babylone.ulb.ac.be/popmusic/) [75] checks for structural stability; Mutation Profiling (http://profile.mutdb.org/) [76] predicts effects of amino acid substitutions, whereas, the structure-based approach elucidates the altered phenotype caused by the protein by predicting effects on its 3D structure [72,77], and its major drawback is restrictive data availability as structural information is not yet available for many proteins [72]. PolyPhen-2 (http:// 
genetics.bwh.harvard.edu/pph2/) [78] and SIFT (http:// sift.jcvi.org/) [79] are two sequence based resources for predicting the functional effect of human SNPs under investigation. Polyphen is less dependent on the multiple alignments used as input. If user alignments for a specific dataset are not available for input, then Polyphen could perhaps be preferred for this reason. On the other hand, if own alignments can be produced then SIFT might be preferable since its web interface allows one to specify the alignment. PROVEAN (http://provean.jcvi.org/) [80] is a tool which also takes in frame insertions, deletions and multiple amino acid substitutions into consideration, being more relevant to variation analysis from next generation sequencing projects.

Lately, variants affecting the post translational protein modifications have received attention, in their potential role in disease causation. Post translational modifications can be reversible or irreversible changes made to a protein after its translation, changing its function by changing protein structure and dynamics or by altering a binding site on it, thus playing an important role in signal transduction pathways and modulating a protein's cellular role [81]. Close to 200 post translational modifications have been recently discovered in the human cell, most of which either facilitate binding of a chemical group to a protein or proteolytic cleavage of the protein [82]. When a polymorphism occurs in a post-translational target site, it can invariably result in a host of downstream effects causing disease or its susceptibility. NetPhos (http://www.cbs.dtu.dk/services/ NetPhos/) [83] is a tool which uses artificial neural networks to predict phosphorylation sites in submitted input sequences, determining susceptible regions and facilitating further checks for disease causation. A resource like PROSITE (http://prosite.expasy.org/) [84] can be used to predict the occurrence of these target sites in and near the $\mathrm{SNP}$, and can be used to analyse the functional repercussion of the polymorphism proximity to the motif.

Keeping the wide range of available products in mind, protein prediction tools should be assessed for the method they follow to determine protein structure variations and the functional causal effect. This can be done by reading their respective manuscripts in detail, and to determine if that method suits, and is the best one for the investigation.

\section{Functional SNPs within the non-coding and regulatory regions}

Eukaryotic gene expression involves multiple steps: gene transcription, processing of RNA through splicing mechanisms, translation into a protein product, post-translational modifications and subsequent protein activity. The majority of gene expression regulation takes place within genetic elements modulating it, like enhancers and silencers, TFBS and splicing sites. The intricacies with which sequence variation give rise to gene expression defects have been covered by Wang et al. [58]. Many computational tools have been developed to aid investigation of SNP effects in each of the above stages of gene expression regulation.

a) SNPs in regulatory elements

SNPs within the regulatory elements of the gene can disrupt gene expression by altering TFBS, influencing the strength of enhancers and promoters, making these SNPs of prime importance to be considered for candidate gene association studies [84]. Below, we list tools for general investigation of genomic region for regulatory elements, in order to filter the genomic regions hosting functional SNPs; and then we move to list tools specifically investigating predicted influence of candidate SNPs on any such region.

Putative genetic regulatory elements such as promoter regions, TFBS, CpG islands over-seeing gene expression, along with microRNA binding sites, are extremely crucial locations where a SNP can cause widespread expression variations and potential disease causing effects, perhaps in a tissue specific nature. Examples of such genetic alterations are discussed by Werner et al. [85]. Information on the spread of these regulatory modules can be obtained from previously mentioned regular sequence databases and software like UCSC genome browser, and Pupasuite [71]. An initial DNAase hypersensitivity check from ENCODE (if available for cell type) using the UCSC genome browser can show open and closed chromatin regions to verify the epigenetic context of the locus. Open chromatin regions indicate exposed regulatory sites hosting important functional elements like transcription factors binding sites, enhancers and ncRNAs [34,86]. Such regions which can be very important in de-differentiation diseases like cancer where epigenetic aberrations are frequent and could have a potential causative nature [87].

Analysis of the regulatory regions involves starting at a few well known websites to find TFBS such as TFBIND (http://tfbind.hgc.jp/) [88], MatInspector (http://www.genomatix.de/matinspector.html) [89], TFSEARCH (http://www.cbrc.jp/research/db/TFSEA RCH.html) [90], MAPPER (http://bio.chip.org/mapper) [91] and also is-rSNP (http://www.genomics.csse. unimelb.edu.au/product-is-rSNP.php) [92] and Regu lomeDB (http://www.regulomedb.org/index) [93], which scans SNP sites for significant potential regulatory elements such as transcription factor binding and histone modifications. FunciSNP, a recent tool available at http://bioconductor.org/ which is itself a rich source of multi-purpose bioinformatic tools, takes into account chromatin features along 
with tag SNP and linked SNPs from the 1000 genomes project to spew out functionally important SNPs specific to non-coding regions [94]. A rare variant rs183373024, was recently explored using FunciSNP and implicated in prostate cancer risk based on its position in a transcription factor occupied region; disrupting a FoxA1 binding site at 8q24 [95].

In certain hormone mediated diseases such as cancer, hormone response elements have causal relations with aberrant hormonal modulations [96]. Thus, promoter regions of candidate genes can be analysed by tools like Dragon ERE Finder (http://datam.i2r.a-star.edu. sg/ereV3/index.html) [97] and JASPER (http://jaspar. genereg.net/) [98] to characterise for the presence of putative estrogen and androgen response elements (EREs and AREs) respectively. CISTER (http://zlab.bu. edu/ mfrith/cister.shtml) [99] can be used to check for both elements. These tools and strategy were used by Batra et al. [25] for a similar purpose.

A recent class of powerful functional elements, which play an extensive role in the genomic regulation as a part of epigenetic mechanisms in the cell, are microRNAs [100]. Their deregulation has been implicated in various diseases like Cancer, Schizophrenia and Autism [101-103]. SNPs lying in miRNA binding regions and interfering with its regulatory function, also called MiRSNPs have also been reported to be associated with risk and with drug resistance in some instances [101]. mirBase (http://www.mirbase.org/) [104] is a microRNA Database which finds targets predicted by microCosm, TargetScan and Pictar [105]. Mirsnpscore (http://www.bigr.medisin.ntnu. no/mirsnpscore/) [106] is a database of SNPs predicted to influence microRNA efficacy by mapping potential causative SNPs to microRNA target sites. MirSNP (http://cmbi.bjmu.edu.cn/mirsnp) [107] provides a database of SNPs which are predicted to enhance/create or decrease/break a miRNA-mRNA binding site. Another tool to find microRNA targets is microRNA.org (http://www.microrna. org/) [108], which also provides experimentally observed gene expression patterns. Two available and well recommended resources for miRSNP information and functional effect prediction in diseases are PolymiRTS database (http://compbio. uthsc.edu/miRSNP/) [109] and Patrocles (http:// www.patrocles.org/) [110]. A comparative strategy of using more than one web tool can increase scope of analysis and circumvent technical drawbacks of the individual tools.

b) eQTL

Expression quantitative trait loci (eQTL) mapping is a technique which uses results from two high throughput techniques, i.e. genome wide gene expre- ssion analysis and the GWAS to define an association between a particular genomic loci variant with a changed gene expression pattern, thus, attributing specific genetic regulatory roles to candidate SNPs in the gene locus [111-114]. cis acting eQTLs are those located near the target genes and have a direct influence on its gene regulation, whereas trans acting eQTLs are located away from the target region and show an indirect remotely regulated gene expression [115].

Software developed towards facilitating mining of genetic expression and variant associations include eQTL Explorer, eQTL Viewer, FastMap and Lirnet. Bioinformatics concepts relating to eQTL have been reviewed in [116]. eQTL Explorer (http://web. bioinformatics.ic.ac.uk/eqtlexplorer/) [117] as an addition to resources provided by previous softwares like WebQTL [118] and QTL Express [119], enables integrated visualization using a Java graphical interfaces; extracts eQTL results from external sources (multiple microarray experiments) and presents them such that they can be compared among each other, and with the pQTL (protein expression) mapped to the genome. eQTL Viewer (http://statgen. ncsu.edu/eQTLViewer/) [120] uses Scalable Vector Graphics for visualisation and carries an added advantage of biological annotations being present dynamically on its interactive mapping results plot. FastMap (http://comptox.unc.edu/fastmap.php) [121], developed in 2009, enables a faster analysis of expression and genotype data by organising SNPs into a hamming distance based tree thus minimizing the number of steps involved. In addition, it provides permutation based significance testing of results. Lirnet (http://www.cs.washington.edu/homes/suinlee/ lirnet/) [122] uses a learning strategy to overcome problems of low population size and correlating SNP effect on gene expression due to large genomic regions being in $\mathrm{LD}$ for any given trait, i.e. it learns the 'regulatory potential' of a SNP through a Bayesian method from its previously known genomic context (such as regulatory networks and features existing and relevant to the gene) and gives an estimate of likelihood of effecting gene expression.

\section{Conclusion}

Recent advances in high-throughput experimental technologies like whole-genome gene expression profiling, the genome wide association studies (GWAS), next generation DNA, RNA sequencing and CHIP-seq scan the genome for disease associated genetic variants and add knowledge to gene function, regulation, SNP prioritisation resources [123,124]. They provide extensive whole genome data and high coverage genomic, transcriptomic, 
epigenomic, and proteomic information in numerous cell types, classifying tissue specific behaviour, interactions and cell functioning $[124,125]$. In present day context, candidate gene studies can utilize the current knowledge resources made available by these initiatives to further discovery, and validating these interactions to uncover a myriad of susceptible disease associations. Applying the candidate gene approach to next generation data is bound to give rich dividends in terms of elucidation of complex disease mechanisms, better prognosis and diagnosis of patients in a short time, and in an efficient way.

\section{Additional files}

Additional file 1: Table S1. List of useful web-tools for candidate gene selection and SNP mapping [5,6,10-21,23,24,27-33,37,39-46,50-55,62].

Additional file 2: Table S2. SNP Effects and functional analysis $[28,62,65-71,75,76,78-80,83,84,88-94,97-99,105-110,117,120-122,126-139]$

\section{Competing interests}

The authors declare that they have no competing interests.

\section{Authors' contributions}

$\mathrm{JB}$ conceived the idea. RP collated the information on all in silico tools and associated websites with input from JB. RP and JB wrote the manuscript. JAC critically reviewed the manuscript. All authors read and approved the final manuscript.

\section{Acknowledgements}

The current work is supported by NHMRC Grant \#1050742; NHMRC Grant \#1009458; NHMRC Principal Research Fellowship (J.A. Clements) and NHMRC Early Career Fellowships (J. Batra). We acknowledge Amanda Spurdle for her review of the manuscript and valuable feedback.

Received: 7 November 2012 Accepted: 15 April 2013

Published: 9 May 2013

\section{References}

1. Kwon JM, Goate AM: The candidate gene approach. Alcohol Res Health 2000, 24(3):164-168

2. Collins FS, Guyer MS, Chakravarti A: Variations on a theme: cataloging human DNA sequence variation. Science 1997, 278(5343):1580-1581.

3. Peters BJM, Rodin AS, De Boer A, Maitland-van der Zee A-H: Methodological and statistical issues in pharmacogenomics. J Pharm Pharmacol 2010, 62(2):161-166.

4. Burdick KE, DeRosse P, Kane JM, Lencz T, Malhotra AK: Genetic variation in the MET proto-oncogene is associated with schizophrenia and general cognitive ability. Am J Psychiatry 2010, 167(4):436-443.

5. Gibbs RA, Belmont JW, Hardenbol P, Willis TD, Yu F, Yang H, Ch'ang L-Y, Huang W, Liu B, Shen Y: The international HapMap project. Nature 2003, 426(6968):789-796.

6. NatureAn integrated map of genetic variation from 1,092 human genomes. 2012, 491(7422):56-65.

7. Pharoah PDP, Dunning AM, Ponder BAJ, Easton DF: Association studies fo finding cancer-susceptibility genetic variants. Nat Rev Cancer 2004, 4(11):850-860.

8. Braem MGM, Schouten $\amalg$, Peeters PHM, den Brandt PA, Onland-Moret NC: Genetic susceptibility to sporadic ovarian cancer: A systematic review. Biochimica et Biophysica Acta (BBA) - Rev Cancer 2011, 1816(2):132-146.

9. Tabor HK, Risch NJ, Myers RM: Candidate-gene approaches for studying complex genetic traits: practical considerations. Nat Rev Genet 2002, 3(5):391-397.

10. Fernández J, Hoffmann R, Valencia A: iHOP Web Services Family. In Bioinformatics for personalized medicine. Edited by Freitas A, Navarro A. Berlin Heidelberg: Springer; 2012:102-107. vol. 6620.
11. Hokamp K, Wolfe KH: PubCrawler: keeping up comfortably with PubMed and GenBank. Nucleic Acids Res 2004, 32(suppl 2):W16-W19.

12. Mastellos D, Andronis C, Persidis A, Lambris JD: Novel biological networks modulated by complement. Clin Immunol 2005, 115(3):225-235.

13. Croft D, O'Kelly G, Wu G, Haw R, Gillespie M, Matthews L, Caudy M, Garapati P, Gopinath $G$, Jassal B, et al: Reactome: a database of reactions, pathways and biological processes. Nucleic Acids Res 2011, 39(suppl 1):D691-D697.

14. Frisch $M$, Klocke $B$, Haltmeier $M$, Frech $K$ : Litlnspector: literature and signal transduction pathway mining in PubMed abstracts. Nucleic Acids Res 2009, 37(suppl 2):W135-W140.

15. Kandasamy K, Mohan SS, Raju R, Keerthikumar S, Kumar G, Venugopal A, Telikicherla D, Navarro JD, Mathivanan S, Pecquet C, et al: NetPath: a public resource of curated signal transduction pathways. Genome Biol 2010, 11(1):R3.

16. Haibe-Kains B, Olsen C, Djebbari A, Bontempi G, Correll M, Bouton C, Quackenbush J: Predictive networks: a flexible, open source, web application for integration and analysis of human gene networks. Nucleic Acids Res 2012, 40(D1):D866-D875.

17. Chen J, Bardes EE, Aronow BJ, Jegga AG: ToppGene Suite for gene list enrichment analysis and candidate gene prioritization. Nucleic Acids Res 2009, 37(suppl 2):W305-W311.

18. Tranchevent L-C, Capdevila FB, Nitsch D, De Moor B, De Causmaecker P, Moreau Y: A guide to web tools to prioritize candidate genes. Brief Bioinform 2011, 12(1):22-32.

19. Amberger J, Bocchini C, Hamosh A: A new face and new challenges for online Mendelian inheritance in man (OMIM $\left.{ }^{\oplus}\right)$. Hum Mutat 2011 32(5):564-567.

20. Radivojac P, Peng K, Clark WT, Peters BJ, Mohan A, Boyle SM, Mooney SD: An integrated approach to inferring gene-disease associations in humans. Proteins: Structure, Function, and Bioinformatics 2008, 72(3):1030-1037.

21. Rhodes DR, Kalyana-Sundaram S, Mahavisno V, Varambally $R$, Yu J, Briggs BB, Barrette TR, Anstet MJ, Kincead-Beal C, Kulkarni P, et al: Oncomine 3.0: genes, pathways, and networks in a collection of 18,000 cancer gene expression Profiles1. New York (NY): Neoplasia Press Inc; 2007.

22. Hu P, Bader G, Wigle DA, Emili A: Computational prediction of cancergene function. Nat Rev Cancer 2007, 7(1):23-34.

23. Wheeler DL, Barrett T, Benson DA, Bryant SH, Canese K, Church DM, DiCuccio M, Edgar R, Federhen S, Helmberg W, et al: Database resources of the national center for biotechnology information. Nucleic Acids Res 2005 , 33(suppl 1):D39-D45

24. Hubbard TJP, Aken BL, Ayling S, Ballester B, Beal K, Bragin E, Brent S, Chen Y, Clapham P, Clarke L, et al: Ensembl 2009. Nucleic Acids Res 2009, 37(suppl 1):D690-D697.

25. Batra J, Nagle C, O'Mara T, Higgins M, Dong Y, Tan O, Lose F, Skeie L, Srinivasan S, Bolton K, et al: A Kallikrein 15 (KLK15) single nucleotide polymorphism located close to a novel exon shows evidence of association with poor ovarian cancer survival. BMC Cancer 2011, 11(1):119.

26. Narla G, DiFeo A, Reeves HL, Schaid DJ, Hirshfeld J, Hod E, Katz A, Isaacs WB, Hebbring S, Komiya A, et al: A Germline DNA polymorphism enhances alternative splicing of the KLF6 tumor suppressor gene and is associated with increased prostate cancer risk. Cancer Res 2005, 65(4):1213-1222.

27. Thierry-Mieg D, Thierry-Mieg J: AceView: a comprehensive cDNAsupported gene and transcripts annotation. Genome Biol 2006, 7(Suppl 1):S12

28. Kuhn RM, Karolchik D, Zweig AS, Wang T, Smith KE, Rosenbloom KR, Rhead B, Raney BJ, Pohl A, Pheasant M, et al: The UCSC genome browser database: update 2009. Nucleic Acids Res 2009, 37(suppl 1):D755-D761.

29. The EPC: A User's guide to the encyclopedia of DNA elements (ENCODE). PLoS Biol 2011, 9(4):e1001046.

30. Frazer KA, Pachter L, Poliakov A, Rubin EM, Dubchak I: VISTA: computational tools for comparative genomics. Nucleic Acids Res 2004, 32(suppl 2):W273-W279.

31. Visel A, Minovitsky S, Dubchak I, Pennacchio LA: VISTA enhancer browsera database of tissue-specific human enhancers. Nucleic Acids Res 2007 35(suppl 1):D88-D92.

32. Lukashin I, Novichkov P, Boffelli D, Paciorkowski AR, Minovitsky S, Yang S, Dubchak I: VISTA region viewer (RViewer) - a computational system for prioritizing genomic intervals for biomedical studies. Bioinformatics 2011, 27(18):2595-2597.

33. Chen Y-H, Liu C-K, Chang S-C, Lin Y-J, Tsai M-F, Chen Y-T, Yao A GenoWatch: a disease gene mining browser for association study. Nucleic Acids Res 2008, 36(suppl 2):W336-W340. 
34. Mooney SD, Krishnan VG, Evani US: Bioinformatic tools for identifying disease gene and SNP candidates. In Genetic Variation. Edited by Barnes MR, Breen G. Humana Press; 2010:307-319. vol. 628

35. Batra J, O'Mara T, Patnala R, Lose F, Clements JA: Genetic polymorphisms in the human tissue kallikrein (KLK) locus and their implication in various malignant and non-malignant diseases. Biol Chem 2012, 393:1365.

36. Coassin S, Brandstätter A, Kronenberg F: Lost in the space of bioinformatic tools: a constantly updated survival guide for genetic epidemiology. The GenEpi toolbox. Atherosclerosis 2010, 209(2):321-335.

37. Sherry ST, Ward M-H, Kholodov M, Baker J, Phan L, Smigielski EM, Sirotkin K: dbSNP: the NCBI database of genetic variation. Nucleic Acids Res 2001 29(1):308-311.

38. Church DM, Lappalainen I, Sneddon TP, Hinton J, Maguire M, Lopez J, Garner J, Paschall J, DiCuccio M, Yaschenko E, et al: Public data archives for genomic structural variation. Nat Genet 2010, 42(10):813-814.

39. Chen K, MCLellan MD, Ding L, Wendl MC, Kasai Y, Wilson RK, Mardis ER: PolyScan: an automatic indel and SNP detection approach to the analysis of human resequencing data. Genome Res 2007, 17(5):659-666.

40. Saccone SF, Quan J, Jones PL: BioQ: tracing experimental origins in public genomic databases using a novel data provenance model. Bioinformatics 2012, 28(8):1189-1191.

41. Saccone SF, Quan J, Mehta G, Bolze R, Thomas P, Deelman E, Tischfield JA, Rice JP: New tools and methods for direct programmatic access to the dbSNP relational database. Nucleic Acids Res 2011, 39(suppl 1):D901-D907.

42. Riva A, Kohane IS: SNPper: retrieval and analysis of human SNPs. Bioinformatics 2002, 18(12):1681-1685.

43. Andersen MC, Engström PG, Lithwick S, Arenillas D, Eriksson P, Lenhard B, Wasserman WW, Odeberg J: In silico detection of sequence variations modifying transcriptional regulation. PLoS Comput Biol 2008, 4(1):e5.

44. Packer BR, Yeager M, Staats B, Welch R, Crenshaw A, Kiley M, Eckert A, Beerman M, Miller E, Bergen A, et al: SNP500Cancer: a public resource for sequence validation and assay development for genetic variation in candidate genes. Nucleic Acids Res 2004, 32(suppl 1):D528-D532.

45. Wang K, Li M, Hakonarson H: ANNOVAR: functional annotation of genetic variants from high-throughput sequencing data. Nucleic Acids Res 2010, 38(16):e164

46. Xu Z, Taylor JA: SNPinfo: integrating GWAS and candidate gene information into functional SNP selection for genetic association studies. Nucleic Acids Res 2009, 37(suppl 2):W600-W605

47. Weiss KM, Clark AG: Linkage disequilibrium and the mapping of complex human traits. Trends in Genetics 2002, 18(1):19-24.

48. Ardlie $K G$, Kruglyak $L$, Seielstad M: Patterns of linkage disequilibrium in the human genome. Nat Rev Genet 2002, 3(4):299-309.

49. Slatkin M: Linkage disequilibrium [mdash] understanding the evolutionary past and mapping the medical future. Nat Rev Genet 2008 9(6):477-485.

50. Pallejà A, Horn H, Eliasson S, Jensen LJ: DistiLD Database: diseases and traits in linkage disequilibrium blocks. Nucleic Acids Res 2012, 40(D1):D1036-D1040.

51. Lawrence R, Day-Williams A, Mott R, Broxholme J, Cardon L, Zeggini E: GLIDERS - A web-based search engine for genome-wide linkage disequilibrium between HapMap SNPs. BMC Bioinforma 2009, 10(1):367.

52. Yoo J, Lee Y, Kim Y, Rha S, Kim Y: SNPAnalyzer 2.0: a web-based integrated workbench for linkage disequilibrium analysis and association analysis. BMC Bioinforma 2008, 9(1):290.

53. Johnson AD, Handsaker RE, Pulit SL, Nizzari MM, O'Donnell CJ, de Bakker PIW: SNAP: a web-based tool for identification and annotation of proxy SNPs using HapMap. Bioinformatics 2008, 24(24):2938-2939.

54. A compilation of some available software for linkage disequilibrium analysis. http://www.genes.org.uk/software/LD-software.shtml.

55. Barrett JC, Fry B, Maller J, Daly MJ: Haploview: analysis and visualization of LD and haplotype maps. Bioinformatics 2005, 21(2):263-265.

56. Jackson DG, Healy MD, Davison DB: Binformatics: not just for sequences anymore. BIOSILICO 2003, 1(3):103-111.

57. Maurano MT, Humbert R, Rynes E, Thurman RE, Haugen E, Wang $H$, Reynolds AP, Sandstrom R, Qu H, Brody J, et al: Systematic localization of common disease-associated variation in regulatory DNA. Science 2012, 337(6099):1190-1195.

58. Wang X, Tomso DJ, Liu X, Bell DA: Single nucleotide polymorphism in transcriptional regulatory regions and expression of environmentally responsive genes. Toxicol Appl Pharmacol 2005, 207(2, Supplement):84-90.
59. Prokunina L, Alarcón-Riquelme ME: Regulatory SNPs in complex diseases: their identification and functional validation. Expert Reviews in Molecular Medicine 2004, 6(10):1-15.

60. GuhaThakurta D, Xie T, Anand M, Edwards S, Li G, Wang S, Schadt E: Cisregulatory variations: a study of SNPs around genes showing cis-linkage in segregating mouse populations. BMC Genomics 2006, 7(1):235.

61. Muinos-Gimeno M, Montfort M, Bayes M, Estivill X, Espinosa-Parrilla Y: Design and evaluation of a panel of single-nucleotide polymorphisms in microRNA genomic regions for association studies in human disease. Eur J Hum Genet 2009, 18(2):218-226.

62. McLaren W, Pritchard B, Rios D, Chen Y, Flicek P, Cunningham F: Deriving the consequences of genomic variants with the Ensembl API and SNP Effect Predictor. Bioinformatics 2010, 26(16):2069-2070.

63. Cooper GM, Shendure J: Needles in stacks of needles: finding diseasecausal variants in a wealth of genomic data. Nat Rev Genet 2011, 12(9):628-640

64. Jiang L, Zhang C, Li Y, Yu X, Zheng J, Zou P, Li Y, Bin X, Lu J, Zhou Y: A non-synonymous polymorphism Thr115Met in the EpCAM gene is associated with an increased risk of breast cancer in Chinese population. Breast Cancer Res Treat 2011, 126(2):487-495

65. Yue P, Melamud E, Moult J: SNPs3D: candidate gene and SNP selection for association studies. BMC Bioinformatics 2006, 7(1):1-15.

66. Ryan M, Diekhans M, Lien S, Liu Y, Karchin R: LS-SNP/PDB: annotated nonsynonymous SNPs mapped to Protein Data Bank structures. Bioinformatics 2009, 25(11):1431-1432.

67. Pieper U, Webb BM, Barkan DT, Schneidman-Duhovny D, Schlessinger A, Braberg H, Yang Z, Meng EC, Pettersen EF, Huang CC, et al: ModBase, a database of annotated comparative protein structure models, and associated resources. Nucleic Acids Res 2011, 39(suppl 1):D465-D474.

68. Jegga AG, Gowrisankar S, Chen J, Aronow BJ: PolyDoms: a whole genome database for the identification of non-synonymous coding SNPs with the potential to impact disease. Nucleic Acids Res 2007, 35(suppl 1):D700-D706.

69. Consortium TU: Reorganizing the protein space at the universal protein resource (UniProt). Nucleic Acids Res 2012, 40(D1):D71-D75.

70. De Baets G, Van Durme J, Reumers J, Maurer-Stroh S, Vanhee P, Dopazo J, Schymkowitz J, Rousseau F: SNPeffect 4.0: on-line prediction of molecular and structural effects of protein-coding variants. Nucleic Acids Res 2012, 40(D1):D935-D939.

71. Conde L, Vaquerizas JM, Dopazo H, Arbiza L, Reumers J, Rousseau F, Schymkowitz J, Dopazo J: PupaSuite: finding functional single nucleotide polymorphisms for large-scale genotyping purposes. Nucleic Acids Res 2006, 34(suppl 2):W621-W625.

72. Mah JTL, Low ESH, Lee E: In silico SNP analysis and bioinformatics tools: a review of the state of the art to aid drug discovery. Drug Discov Today 2011, 16(17-18):800-809.

73. Mah JTL, Low ESH, Lee E: In silico SNP analysis and bioinformatics tools: a review of the state of the art to aid drug discovery. Drug discovery today 2011, 16(17):800-809.

74. Miller MP, Kumar S: Understanding human disease mutations through the use of interspecific genetic variation. Hum Mol Genet 2001, 10(21):2319-2328.

75. Kwasigroch JM, Gilis D, Dehouck Y, Rooman M: PoPMuSiC, rationally designing point mutations in protein structures. Bioinformatics 2002 18(12):1701-1702.

76. Mort M, Evani US, Krishnan VG, Kamati KK, Baenziger PH, Bagchi A, Peters BJ, Sathyesh R, Li B, Sun Y, et al: In silico functional profiling of human disease-associated and polymorphic amino acid substitutions. Hum Mutat 2010, 31(3):335-346.

77. Sunyaev S, Ramensky V, Bork P: Towards a structural basis of human nonsynonymous single nucleotide polymorphisms. Trends in Genetics 2000 16(5):198-200.

78. Adzhubei IA, Schmidt S, Peshkin L, Ramensky VE, Gerasimova A, Bork P, Kondrashov AS, Sunyaev SR: A method and server for predicting damaging missense mutations. Nat Meth 2010, 7(4):248-249.

79. Kumar P, Henikoff S, Ng PC: Predicting the effects of coding nonsynonymous variants on protein function using the SIFT algorithm. Nat Protocols 2009, 4(8):1073-1081.

80. Choi Y, Sims GE, Murphy S, Miller JR, Chan AP: Predicting the functional effect of amino acid substitutions and Indels. PLOS ONE 2012, 7(10):e46688. 
81. Li S, lakoucheva LM, Mooney SD, Radivojac P: Loss of post-translational modification sites in disease. In Pac Symp Biocomput. World Scientific: 2010:337-347.

82. Mann M, Jensen ON: Proteomic analysis of post-translational modifications. Nat Biotech 2003, 21(3):255-261

83. Blom N, Gammeltoft S, Brunak S: Sequence and structure-based prediction of eukaryotic protein phosphorylation sites. J Mol Biol 1999, 294(5):1351-1362.

84. Sigrist CJA, Cerutti L, de Castro E, Langendijk-Genevaux PS, Bulliard V, Bairoch A Hulo N: PROSITE, a protein domain database for functional characterization and annotation. Nucleic Acids Res 2010, 38(suppl 1):D161-D166.

85. Werner T: Functional in silico analysis of Non-coding SNPS, Bioinformatics for geneticists. Chichester, UK: John Wiley \& Sons, Ltd; 2003:273-287.

86. Boyle AP, Davis S, Shulha HP, Meltzer P, Margulies EH, Weng Z, Furey TS, Crawford GE: High-resolution mapping and characterization of open chromatin across the genome. Cell 2008, 132(2):311-322.

87. Gaspar-Maia A, Alajem A, Meshorer E, Ramalho-Santos M: Open chromatin in pluripotency and reprogramming. Nat Rev Mol Cell Biol 2011, 12(1):36-47.

88. Tsunoda T, Takagi T: Estimating transcription factor bindability on DNA. Bioinformatics 1999, 15(7):622-630.

89. Cartharius K, Frech K, Grote K, Klocke B, Haltmeier M, Klingenhoff A, Frisch M, Bayerlein M, Werner T: MatInspector and beyond: promoter analysis based on transcription factor binding sites. Bioinformatics 2005, 21(13):2933-2942.

90. Heinemeyer $\mathrm{T}$, Wingender $\mathrm{E}$, Reuter I, Hermjakob H, Kel AE, Kel OV, Ignatieva EV, Ananko EA, Podkolodnaya OA, Kolpakov FA, et al: Databases on transcriptional regulation: TRANSFAC, TRRD and COMPEL. Nucleic Acids Res 1998, 26(1):362-367.

91. Marinescu VD, Kohane IS, Riva A: The MAPPER database: a multi-genome catalog of putative transcription factor binding sites. Nucleic Acids Res 2005, 33(suppl 1):D91-D97

92. Macintyre G, Bailey J, Haviv I, Kowalczyk A: Is-rSNP: a novel technique for in silico regulatory SNP detection. Bioinformatics 2010, 26(18):i524-i530.

93. Boyle AP, Hong EL, Hariharan M, Cheng Y, Schaub MA, Kasowski M, Karczewski KJ, Park J, Hitz BC, Weng S, et al: Annotation of functional variation in personal genomes using RegulomeDB. Genome Res 2012, 22(9):1790-1797.

94. Coetzee SG, Rhie SK, Berman BP, Coetzee GA, Noushmehr H: FunciSNP: an $\mathrm{R} /$ bioconductor tool integrating functional non-coding data sets with genetic association studies to identify candidate regulatory SNPs. Nucleic Acids Res 2012, 40(18):e139.

95. Hazelett DJ, Coetzee SG, Coetzee GA: A rare variant, which destroys a FoxA1 site at $8 \mathrm{q} 24$, is associated with prostate cancer risk. Cell Cycle 2013, 12(2):379-380.

96. Risch HA: Hormonal etiology of epithelial ovarian cancer, with a hypothesis concerning the role of androgens and progesterone. J Natl Cancer Inst 1998, 90(23):1774-1786.

97. Bajic VB, Tan SL, Chong A, Tang S, Ström A, Gustafsson J-Å, Lin C-Y, Liu ET: Dragon ERE Finder version 2: a tool for accurate detection and analysis of estrogen response elements in vertebrate genomes. Nucleic Acids Res 2003, 31(13):3605-3607.

98. Portales-Casamar E, Thongjuea S, Kwon AT, Arenillas D, Zhao X, Valen E, Yusuf D, Lenhard B, Wasserman WW, Sandelin A: JASPAR 2010: the greatly expanded open-access database of transcription factor binding profiles. Nucleic Acids Res 2010, 38(suppl 1):D105-D110.

99. Frith MC, Hansen U, Weng Z: Detection of cis -element clusters in higher eukaryotic DNA. Bioinform 2001, 17(10):878-889.

100. Freedman ML, Monteiro ANA, Gayther SA, Coetzee GA, Risch A, Plass C, Casey G, De Biasi M, Carlson C, Duggan D, et al: Principles for the postGWAS functional characterization of cancer risk loci. Nat Genet 2011, 43(6):513-518.

101. Mishra PJ, Mishra PJ, Banerjee D, Bertino JR: MiRSNPs or MiRpolymorphisms, new players in microRNA mediated regulation of the cell: Introducing microRNA pharmacogenomics. Cell Cycle 2008, 7(7):853-858.

102. Sun G, Yan J, Noltner K, Feng J, Li H, Sarkis DA, Sommer SS, Rossi JJ: SNPs in human miRNA genes affect biogenesis and function. RNA 2009, 15(9):1640-1651.

103. Davis-Dusenbery BN, Hata A: MicroRNA in cancer: the involvement of aberrant MicroRNA biogenesis regulatory pathways. Genes \& Cancer 2010, 1(11):1100-1114
104. Betel D, Wilson M, Gabow A, Marks DS, Sander C: The microRNA.org resource: targets and expression. Nucleic Acids Res 2008, 36(suppl 1):D149-D153.

105. Kozomara A, Griffiths-Jones S: miRBase: integrating microRNA annotation and deep-sequencing data. Nucleic Acids Res 2011, 39(suppl 1):D152-D157.

106. Thomas LF, Saito T, Sætrom P: Inferring causative variants in microRNA target sites. Nucleic Acids Res 2011, 39(16):e109.

107. Liu C, Zhang F, Li T, Lu M, Wang L, Yue W, Zhang D: MirSNP, a database of polymorphisms altering miRNA target sites, identifies miRNA-related SNPs in GWAS SNPs and eQTLs. BMC Genom 2012, 13(1):661.

108. Betel D, Koppal A, Agius P, Sander C, Leslie C: Comprehensive modeling of microRNA targets predicts functional non-conserved and non-canonical sites. Genome Biol 2010, 11(8):R90.

109. Ziebarth JD, Bhattacharya A, Chen A, Cui Y: PolymiRTS Database 2.0: linking polymorphisms in microRNA target sites with human diseases and complex traits. Nucleic Acids Res 2012, 40(D1):D216-D221.

110. Hiard S, Charlier C, Coppieters W, Georges M, Baurain D: Patrocles: a database of polymorphic miRNA-mediated gene regulation in vertebrates. Nucleic Acids Res 2010, 38(suppl 1):D640-D651.

111. Gilad Y, Rifkin SA, Pritchard JK: Revealing the architecture of gene regulation: the promise of eQTL studies. Trends Genet 2008, 24(8):408-415.

112. Jansen RC, Nap J-P: Genetical genomics: the added value from segregation. Trends Genet 2001, 17(7):388-391.

113. Jansen RC: Studying complex biological systems using multifactorial perturbation. Nat Rev Genet 2003, 4(2):145-151.

114. Wu C, Delano DL, Mitro N, Su SV, Janes J, McClurg P, Batalov S, Welch GL, Zhang J, Orth AP, et al: Gene Set enrichment in eQTL data identifies novel annotations and pathway regulators. PLoS Genet 2008, 4(5):e1000070

115. Wittkopp PJ: Genomic sources of regulatory variation in cis and in trans. Cell Mol Life Sci 2005, 62(16):1779-1783.

116. Li H, Deng H: Systems genetics, bioinformatics and eQTL mapping. Genetica 2010, 138(9):915-924.

117. Mueller M, Goel A, Thimma M, Dickens NJ, Aitman TJ, Mangion J: eQTL Explorer: integrated mining of combined genetic linkage and expression experiments. Bioinform 2006, 22(4):509-511.

118. Wang J, Williams R, Manly K: WebQTL. Neuroinform 2003, 1(4):299-308.

119. Seaton G, Haley CS, Knott SA, Kearsey M, Visscher PM: QTL Express: mapping quantitative trait loci in simple and complex pedigrees. Bioinform 2002, 18(2):339-340.

120. Zou W, Aylor D, Zeng Z-B: eQTL Viewer: visualizing how sequence variation affects genome-wide transcription. BMC Bioinform 2007, 8(1):7.

121. Gatti DM, Shabalin AA, Lam T-C, Wright FA, Rusyn I, Nobel AB: FastMap: Fast eQTL mapping in homozygous populations. Bioinform 2009, 25(4):482-489.

122. Lee S-I, Dudley AM, Drubin D, Silver PA, Krogan NJ, Pe'er D, Koller D: Learning a prior on regulatory potential from eQTL data. PLOS Genet 2009, 5(1):e1000358.

123. Giacomini KM, Brett CM, Altman RB, Benowitz NL, Dolan ME, Flockhart DA, Johnson JA, Hayes DF, Klein T, Krauss RM, et al: The Pharmacogenetics research network: from SNP discovery to clinical drug response. Clin Pharmacol Ther 2007, 81(3):328-345.

124. Hawkins RD, Hon GC, Ren B: Next-generation genomics: an integrative approach. Nat Rev Genet 2010, 11(7):476-486.

125. Horgan R, Kenny L: 'Omic'technologies: genomics, transcriptomics, proteomics and metabolomics. The Obstetrician \& Gynaecologist 2011 13(3):189-195

126. Cartegni L, Wang J, Zhu Z, Zhang MQ, Krainer AR: ESEfinder: a web resource to identify exonic splicing enhancers. Nucleic Acids Res 2003, 31(13):3568-3571.

127. Smith PJ, Zhang C, Wang J, Chew SL, Zhang MQ, Krainer AR: An increased specificity score matrix for the prediction of SF2/ASF-specific exonic splicing enhancers. Hum Mol Genet 2006, 15(16):2490-2508.

128. Goren A, Ram O, Amit M, Keren H, Lev-Maor G, Vig I, Pupko T, Ast G: Comparative analysis identifies exonic splicing regulatory sequences the complex definition of enhancers and silencers. Mol Cell 2006, 22(6):769-781.

129. Wang Z, Rolish ME, Yeo G, Tung V, Mawson M, Burge CB: Systematic identification and analysis of exonic splicing silencers. Cell 2004 119(6):831-845.

130. Zhang $X H-F$, Chasin LA: Computational definition of sequence motifs governing constitutive exon splicing. Genes Dev 2004, 18(11):1241-1250. 
131. Fairbrother WG, Yeh R-F, Sharp PA, Burge CB: Predictive identification of exonic splicing enhancers in human genes. Science 2002, 297(5583):1007-1013.

132. Desmet F-O, Hamroun D, Lalande M, Collod-Béroud G, Claustres M, Béroud C: Human splicing finder: an online bioinformatics tool to predict splicing signals. Nucleic Acids Res 2009, 37(9):e67.

133. Ok Yang J, Kim W-Y, Bhak J: ssSNPTarget: genome-wide splice-site single nucleotide polymorphism database. Hum Mutat 2009, 30(12):E1010-E1020.

134. Ahmed F, Kumar M, Raghava GPS: Prediction of polyadenylation signals in human DNA sequences using nucleotide frequencies. In Silico Biol 2009, 9(3):135-148.

135. Tabaska JE, Zhang MQ: Detection of polyadenylation signals in human DNA sequences. Gene 1999, 231(1-2):77-86.

136. Zuker M: Mfold web server for nucleic acid folding and hybridization prediction. Nucleic Acids Res 2003, 31(13):3406-3415.

137. Reeder J, Steffen P, Giegerich R: pknotsRG: RNA pseudoknot folding including near-optimal structures and sliding windows. Nucleic Acids Res 2007, 35(suppl 2):W320-W324.

138. Reeder J, Höchsmann M, Rehmsmeier M, Voss B, Giegerich R: Beyond Mfold: Recent advances in RNA bioinformatics. J Biotechnol 2006, 124(1):41-55.

139. Lambert A, Fontaine J-F, Legendre M, Leclerc F, Permal E, Major F, Putzer $H$, Delfour O, Michot B, Gautheret D: The ERPIN server: an interface to profile-based RNA motif identification. Nucleic Acids Res 2004, 32(suppl 2): W160-W165.

doi:10.1186/1471-2156-14-39

Cite this article as: Patnala et al:: Candidate gene association studies: a comprehensive guide to useful in silico tools. BMC Genetics 2013 14:39.

\section{Submit your next manuscript to BioMed Central and take full advantage of:}

- Convenient online submission

- Thorough peer review

- No space constraints or color figure charges

- Immediate publication on acceptance

- Inclusion in PubMed, CAS, Scopus and Google Scholar

- Research which is freely available for redistribution 